\title{
Optimal sensor location and reduced order observer design for distributed process systems
}

Antonio A. Alonso ${ }^{\mathrm{a}, *}$, Ioannis G. Kevrekidis ${ }^{\mathrm{b}}$, Julio R. Banga ${ }^{\mathrm{a}}$, Christos E. Frouzakis ${ }^{\mathrm{b}}$

${ }^{a}$ Process Engineering Group, IIM-CSIC, Eduardo Cabello 6, 36208 Vigo, Spain

${ }^{\mathrm{b}}$ Department of Chemical Engineering, Princeton University, Princeton, NJ, USA

* Corresponding author. Fax: /34-9-86-292762. E-mail address: antonio@iim.csic.es (A.A. Alonso).

Abstract

This paper presents a systematic approach to efficiently reconstruct the infinite dimensional field in distributed process systems from a limited, and usually reduced, number of sensors. To that purpose, two basic tools are employed: on the one hand, a reduced order representation of the system which, based on proper orthogonal decomposition (POD) expansions, captures the most relevant dynamic features of the solution. On the other hand, the selection of the most appropriate type (and number) of measurements by the solution of a max-min optimization problem. These ideas will be illustrated on the problem of field reconstruction for unstable tubular reactors.

Keywords: Distributed process systems; Observer design; Proper orthogonal decomposition; Optimal sensor placement

\section{Introduction}

The operation and control of distributed process systems such as chemical reactors or flow units usually requires precise information on the spatial distribution of the dynamic variables of interest (e.g. temperature, concentrations or fluid velocity fields). Unfortunately, that information is only available through a limited number of possibly expensive sensors (see, for instance, Van der Berg, Hoefsloot, Boelens \& Smilde, 2000). Such limitation justified over the past years a considerable research effort in establishing methods for efficient sensor placement and field reconstruction (Kumar \& Seinfeld, 1978; Harris, Wright \& MacGregor, 1980; Alvarez, Romagnoli \& Stephanopoulos, 1981; Windes, Cinar \& Ray, 1989). Although the need for systematic methods was soon recognized, most techniques relied on exhaustive search over a pre-defined set of candidates as in Keller and Bonvin (1992).However, this approach, valid for a small number of locations, becomes useless when the number of possible location candidates increases, as it is the case, for instance, in flow reconstruction problems (Podvin \& Lumley, 1998). Exceptions to exhaustive search include sub-optimal sequential selection alternatives, successfully applied to place sensors in large space structures (Kammer, 1991). Other interesting approaches include 
those recently developed by Van den Berg et al. (2000), Vande Wouwer, Point, Porteman and Remy (2000) and Antoniades and Christofides (2001a), although limited by the type of dynamic system representation and control scheme employed.

The methodology we propose in this paper has been developed to handle those situations demanding sensor placement neither depend on the particular control structure nor on a particular dynamic model, as this could be poorly understood or unavailable. With this intention, our approach only requires knowledge of a low dimensional linear sub-space capturing the most relevant features of the process operation. On this linear sub-space, the optimal sensor placement will be computed as that which maximizes a criterion directly connected with the quality of the estimation. An efficient guided search algorithm, with optimal convergence properties, is developed to that purpose. We also show that such selection criterion will also result into dynamic observation schemes with fast convergence rates. These ideas will be illustrated on a case study involving concentration and temperature reconstruction from measurements for a tubular reactor operating at an unstable regime.

2. A reduced order representation of distributed dissipative process systems

The class of systems we are considering in this work are those described by sets of quasi-linear partial differential equations of the form:

$\mathrm{u}_{\mathrm{t}}=\mathrm{L}(\mathrm{u})+\mathrm{F}(\mathrm{u})$

where $\mathrm{L}(\cdot)$ denotes a general linear parabolic operator defined on a (possibly 3-dimensional) spatial domain $\mathrm{D}$ with smooth boundary $\mathrm{B}$ and $\mathrm{F}(\mathrm{u})$ is a nonlinear function of $\mathrm{u}$. The solution $\mathrm{u}(\mathrm{x}, \mathrm{t})$ in equation (1), with appropriate initial and boundary conditions, will be referred to as the field with $\mathrm{x}=\left(\mathrm{x}_{1}, \mathrm{x}_{2}, \mathrm{x}_{3}\right)^{\mathrm{T}}$ denoting spatial coordinates. Eq. (1) can be interpreted as an infinite dimensional system on a Hilbert space equipped with inner product and norm:

$$
\langle f, g\rangle=\int_{D} f(x) g(x) d x,\|f\|_{2}^{2}=\langle f, f\rangle
$$

The dissipative nature of this type of systems allows the expansion of the solution $u$, in terms of a convergent series of the form:

$u(x, t)=\sum_{j=1}^{\infty} c_{j}(t) \varphi_{j}(x)$

where the coefficient set $\left\{\mathrm{c}_{\mathrm{j}}(\mathrm{t})\right\} \stackrel{\infty}{j=1}$ collects the time evolution of the field and the set of basis functions $\left\{\varphi_{\mathrm{j}}(\mathrm{x})\right\}^{\infty}{ }^{j=1}$ determines its spatial dependence (Smoller, 1983). Each element $\varphi_{\mathrm{j}}$ of the set can be 
considered as the solution of the following eigenvalue problem:

$\varphi_{j}(x)=\mu_{j} \int_{D} K\left(x, x^{\prime}\right) \varphi_{j}\left(x^{\prime}\right) d x^{\prime}$

where $\mu_{\mathrm{j}}$ is a parameter-eigenvalue-associated with each basis function $\varphi_{\mathrm{j}}$, and $\mathrm{K}\left(\mathrm{x}, \mathrm{x}^{\prime}\right)$ a given kernel. Depending on the structure of the kernel K, different sets will emerge (Alonso, Kevrekidis \& Frouzakis, 2003). Representative examples include the spectral basis or the so-called empirical eigenfunctions (Berkooz, Holmes \& Lumley, 1993; Holmes, Lumley \& Berkooz, 1996), also known as the proper orthogonal decomposition set (POD). The former is obtained by using the Green function associated to the parabolic operator as a kernel (Courant \& Hilbert, 1937) while the latter is associated to the two point correlation kernel:

$K\left(x, x^{\prime}\right)=\lim _{T \rightarrow \infty} \frac{1}{T} \int_{0}^{T} u(x, t) u\left(x^{\prime}, t\right) d t$

where $\mathrm{T}$ is a given time horizon. In both cases, the ordering property of the eigenspectrum $\left\{\mu_{\mathrm{j}}\right\}^{\infty}=1$, allows the selection of a finite low dimensional set of orthonormal functions $\left.\left\{\varphi_{\mathrm{j}}(\mathrm{x})\right\}\right\}^{k}=1$ capturing the most relevant features of the solution (Christofides \& Daoutidis, 1997). In this way, the field (2) can be approximated as a truncated series so that:

$u \rightarrow \sum_{j=1}^{k} c_{j}(t) \varphi_{j}(x)$

In addition, such ordering property makes it possible to define a small positive parameter $\varepsilon<1$ which partitions the dynamic evolution of the c coefficients in (2), into a slow and fast time scales $t$ and $\tau=t / \varepsilon$, respectively. The explicit form of the dynamics can be obtained by Galerking projection - see for instance Christofides and Daoutidis (1997) - of the original equation (1) on the finite set of basis functions so that: $\dot{c}_{s}=A_{s} c_{s}+f_{s}\left(c_{s}, c_{f}\right)$

$\frac{d c_{f}}{d \tau}=\varepsilon A_{f} c_{f}+\varepsilon f_{f}\left(c_{s}, c_{f}\right)$

where $c^{T}=\left(c_{s}^{T}, c_{f}^{T}\right)$ and $\mathrm{A}_{\mathrm{s}}, \mathrm{A}_{\mathrm{f}}$ are square matrices resulting from the projection of the linear operator $\mathrm{L}(\cdot)$. The elements of the vector functions $\left(f_{s}^{T}, f_{f}^{T}\right)^{T}$ are of the form:

$f_{j}=\left\langle\varphi_{j}, F(u)\right\rangle$

In the limit when $\varepsilon \rightarrow 0$, fast modes relax so that $\frac{d c_{f}}{d \tau} \rightarrow 0$. If in addition $\mathrm{c}_{\mathrm{f}} \rightarrow 0$, the solution $\mathrm{u}(\mathrm{x}, \mathrm{t}) \mathrm{can}$ be approximated by a finite number of slow modes $\mathrm{c}_{\mathrm{s}} \in \mathrm{R}^{\mathrm{ns}}$ in (5) so that $\mathrm{u} \rightarrow \mathrm{u}_{\mathrm{s}}$ with $u_{s}=\sum_{j=1}^{n s} c_{j}(t) \varphi_{j}(x)$ 
We will refer to $u_{s}$ as the slow solution. This representation will be employed in the next section to derive dynamic field reconstruction schemes.

\subsection{Construction of the discrete POD set}

In order to comply with the fact that measurements are only available at a finite number of locations, the reduced order representation discussed in the previous section will be constructed on a discrete basis set

$\left\{\phi_{\mathrm{j}}\right\}^{\stackrel{\infty}{j}=1}$. This set will be interpreted as the n-discrete version of the set $\left\{\varphi_{\mathrm{j}}\right\}^{\stackrel{\infty}{j}=1}$ along the spatial coordinates $\mathrm{x}$ in (2), so that $\phi_{\mathrm{j}} \in \mathrm{R}^{\mathrm{n}}$ and ${ }^{\phi_{j}^{T}} \phi_{\mathrm{k}}=\delta_{\mathrm{jk}}$ for every $\mathrm{j}$ and $\mathrm{k}$, with $\delta_{\mathrm{jk}}$ being the Kronecker delta. To that purpose, let us consider a set of data $\mathrm{S}_{1}\left\{\mathrm{u}_{\mathrm{j}}\right\}^{\ell}=1$, obtained either by experiments or simulation. Each element of the set is an n-dimensional vector with components corresponding to the value of the field at particular locations. If several fields are involved in the description, they will be included in the vector sequentially. The set $S_{1}$ is assumed to be representative of the dynamic behavior of the system in the range of initial conditions, parameters, inputs and/or perturbations of interest (Shvartsman \& Kevrekidis, 1998).

Given an integer $\mathrm{k}(\mathrm{k} \leq \mathrm{n})$, we define the $\mathrm{k}$-dimensional set $\mathrm{S}_{\mathrm{k}}=\left\{\phi_{\mathrm{j}}\right\}^{k}=1$, as the set of $\mathrm{k}$ orthonormal vectors on which the average projection of the data set $\mathrm{S}_{1}$ is maximized (equivalently, the k-set which minimizes the average distance to the data). Formally, the elements of this set can be computed as: $\min _{\phi j} \mathrm{~J}\left(\phi_{\mathrm{j}}, \mathrm{u}\right)$

$J\left(\phi_{j}, u\right)=\frac{1}{\ell} \sum_{i=1}^{\ell}\left(u_{i}^{T}-w_{i}^{T}\right)\left(u_{i}-w_{i}\right)+\sum_{j=1}^{k} \lambda_{j}\left(\phi_{j}^{T} \phi_{j}-1\right)$

where $\mathrm{w}_{\mathrm{i}}$ represents the projection of the data $\mathrm{u}_{\mathrm{i}}$ on the span of the $\mathrm{k}$ vectors $\phi_{\mathrm{j}}$ :

$\mathrm{w}_{\mathrm{i}}=\phi \mathrm{c}_{\mathrm{i}}$

with $\phi$ being the matrix:

$\phi=\left[\phi_{1}: \phi_{\mathrm{k}}\right]$

and $c_{i} \in R^{k}$ a vector of coefficients satisfying $c_{i}=\phi^{T} u_{i}$. The solution of the previous optimization problem leads to the following set of equations to be satisfied by each element in $\mathrm{S}_{\mathrm{k}}$ :

$R \phi_{j}=\lambda_{j} \phi_{j}$

$R=\frac{1}{\ell} \sum_{i=1}^{\ell} u_{i} u_{i}^{T}$

Note that the eigenvalue problem (9) with matrix R as in (10) can be considered as the discrete version of the generalized eigenvalue problem (3) with a kernel of the form (4) and $\mu_{j}=1 / \lambda_{j}$. Consequently, the set $\left\{\phi_{j}\right\}_{j}^{k}=1$ can be interpreted as the discrete version of the POD set $\left\{\varphi_{j}\right\}_{j}^{k}=1$. On the other hand, any 
element $u_{i}$ of the data set $S_{1}$ can be expressed in terms of $S_{k}$ as:

$\mathrm{u}_{\mathrm{i}} \phi \mathrm{c}_{\mathrm{i}}=\varepsilon_{\mathrm{i}}$

where $\varepsilon_{\mathrm{i}}$ is an error vector, orthogonal to $\mathrm{S}_{\mathrm{k}}$ that indicates the distance at which each data point lies from the low dimensional projection plane. The average distance of the data set to $\mathrm{S}_{\mathrm{k}}$ is computed as:

$D_{a v}^{2}=\frac{1}{\ell} \sum_{i=1}^{\ell} d_{i}^{2}=\frac{1}{\ell} \sum_{i=1}^{\ell} u_{i}^{T} u_{i}-\sum \lambda_{i}$

Note that for $\mathrm{k}=1, \mathrm{D}_{\mathrm{av}}=0$ and we have that:

$\sum_{i=1}^{\ell} \lambda_{i}=\frac{1}{\ell} \sum_{i=1}^{\ell} u_{i}^{T} u_{i}$

so the eigenvalues $\lambda_{\mathrm{i}}$ provide a measure of how close the data are to the set $\mathrm{S}_{\mathrm{k}}$. The nearer $\mathrm{k}$ is to 1 , the smaller the average distance. Combining (12) and (13) we have the equivalent expression:

$D_{a v}=\left(\sum_{i=k+1}^{\ell} \lambda_{i}\right)^{\frac{1}{2}}$

The selection of the dimension of the low dimensional set is usually done by means of some related measures, known as the fraction of energy captured, or lost by the reduced order description, and defined, respectively, as:

$E=\frac{\sum_{i=1}^{k} \lambda_{i}}{\sum_{i=1}^{\ell} \lambda_{i}}$

$\mathrm{L}=1-\mathrm{E}$

3. Observer design for field reconstruction

Let us consider a set of $\mathrm{k}$ orthonormal and $\mathrm{n}$-dimensional vectors $\mathrm{S}_{\mathrm{k}}\left\{\phi_{\mathrm{i}}\right\}^{k}=1$, representative of a given system (1) in the sense described in the previous section. In addition, let us define the operator $\mathrm{P}_{\mathrm{m}}$ as that which projects any vector $\mathrm{v} \in \mathrm{R}^{\mathrm{n}}$ on $\mathrm{m}$ of its $\mathrm{n}$ coordinates (the measurement sub-space). Formally, $\mathrm{P}_{\mathrm{m}}$ will be expressed as a $\mathrm{R}^{\mathrm{m} \times \mathrm{n}}$ matrix with rows being $\mathrm{n}$-dimensional unit vectors $\mathrm{e}^{j}$, for $\mathrm{j}=1, \ldots, \mathrm{n}$, so that $\left(e_{j}^{i}\right)^{T} v=v_{i}$ computes the j-measurement at spatial location i. Then, by using (7)-(8) we have that:

$\mathrm{V}_{\mathrm{m}}=\mathrm{Q}^{\mathrm{T}} \mathrm{V}$

$\mathrm{Q}^{\mathrm{T}}=\mathrm{P}_{\mathrm{m}} \phi$

If $\mathrm{v}_{\mathrm{m}}$ is a vector of measurements, and $\mathrm{v}$ an element of the data set $\mathrm{S}_{\mathrm{l}}$, the estimation problem consists of reconstructing the remaining nm components of $\mathrm{v}$ from the available measurements. By making use of the reduced order representation described in the previous section, the field can be reconstructed by computing 
$\hat{c}$ estimates obtained from a set of differential equations:

$\hat{c}_{t}=A \hat{c}+f(\hat{c})+\Omega\left(v_{m}-Q^{T} \hat{c}\right)$

$\hat{u}=\phi \hat{c}$

where $\Omega$ is a gain matrix which determines the (temporal) rate at which the estimate $\hat{c} \rightarrow c$. Equation (17), known as a Luenberger observer, is nothing but a replica of system (5) with c replacing $\mathrm{c}_{\mathrm{s}}$ plus an extraterm which, by computing the difference between the measurements and the state estimates, drives the estimated state $\hat{c}$ towards the "true" state c. The following result, adapted to nonlinear systems of the form (5) from a standard theorem on observability of linear systems (Rugh, 1993), will relate the rate of convergence to the relative positions of the low dimensional sub-space $S_{k}$ and the measurement sub-space (determined by the operator $\mathrm{P}_{\mathrm{m}}$ ). This result will provide a useful criterion to select the appropriate sensor placement.

Theorem 1. Let the system:

$c_{t}=A c+f(c)$

$\mathrm{v}_{\mathrm{m}}=\mathrm{Q}^{\mathrm{T}} \mathrm{c}$

be such that: $\|\mathrm{A}\|=\max _{\|\mathrm{x}\|=1}\|\mathrm{Ax}\|=\alpha \mathrm{m},\left\|\mathrm{f}\left(\mathrm{c}_{1}\right)-\mathrm{f}\left(\mathrm{c}_{2}\right)\right\| \leq \beta\left\|\mathrm{c}_{1}-\mathrm{c}_{2}\right\|$ and: $\lambda_{\mathrm{u}} \mathrm{I} \geq \mathrm{QQ} \mathrm{Q}^{\mathrm{T}} \geq \lambda_{\mathrm{I}} \mathrm{I}$ (19)

The observer (17) with $\Omega=\mathrm{P}_{-}{ }^{1} \mathrm{Q}$, and $\mathrm{P}$ being a symmetric, positive definite matrix satisfying:

$(\mathrm{A}+\alpha \mathrm{I})^{\mathrm{T}} \mathrm{P}+\mathrm{P}(\mathrm{A}+\alpha \mathrm{I})=\mathrm{QQ}^{\mathrm{T}}$

with $0<\eta<1$ and $\alpha>\alpha_{\mathrm{m}}+\frac{\beta \lambda_{u}}{(1-\eta) \lambda_{l}}$, will make the states $\hat{c} \rightarrow c$ at an exponential rate proportional to $\eta \lambda_{\mathrm{l}}$.

Proof. First, note that the error between the estimates and the states $\varepsilon=c-\hat{c}$ evolves in time as:

$\varepsilon_{\mathrm{t}}=\left(\mathrm{A}-\mathrm{P}^{-1} \mathrm{QQ}^{\mathrm{T}}\right) \varepsilon+\mathrm{f}(\mathrm{c})-f(\hat{c})$

The Proof is made in the following steps.

1. Construct a symmetric matrix $P$ as:

$P=\int_{0}^{\infty} e^{-(A+\alpha I)^{T} t} Q Q^{T} e^{-(A+\alpha I) t} d t$

The matrix $P$ is positive definite since $Q^{T}{ }^{T}$ satisfies (19). It is also bounded since the differential system $y_{t}$ $=(\mathrm{A}+\alpha \mathrm{I}) \mathrm{y}$ is exponentially stable for any $\alpha>\alpha \mathrm{m}$. In fact, given an arbitrary vector $\mathrm{y}_{0}$, the quadratic form $y_{0}^{T} P y_{0}$ is bounded as:

$y_{0}^{T} P y_{0} \leq \frac{\lambda_{u}}{2\left(\alpha-\alpha_{m}\right)} y_{0}^{T} y_{0}$ 
By construction, $\mathrm{P}$ also satisfies $(20)$. Let $\mathrm{H}(\mathrm{t})=\mathrm{e}^{-(\mathrm{A}+\alpha \mathrm{I}) \mathrm{Tt}} \mathrm{QQ} \mathrm{Q}^{\mathrm{T}} \mathrm{e}^{-(\mathrm{A}+\alpha \mathrm{I}) \mathrm{t}}$, differentiating $\mathrm{H}$ and integrating over time in the interval $(0, \infty)$, we get:

$\mathrm{dH}=-(\mathrm{A}+\alpha \mathrm{I})^{\mathrm{T}} \mathrm{H}(\mathrm{t})-\mathrm{H}(\mathrm{t})(\mathrm{A}+\alpha \mathrm{I})-\mathrm{H}(0)=-(\mathrm{A}+\alpha \mathrm{I})^{\mathrm{T}} \mathrm{P}-\mathrm{P}(\mathrm{A}+\alpha \mathrm{I})$

and

$(\mathrm{A}+\alpha \mathrm{I})^{\mathrm{T}} \mathrm{P}+\mathrm{P}(\mathrm{A}+\alpha \mathrm{I})=\mathrm{QQ}^{\mathrm{T}}$

2. Construct a Lyapunov function $\mathrm{V}=\varepsilon^{\mathrm{T}} \mathrm{P} \varepsilon$ and compute its time derivative:

$\mathrm{V}_{\mathrm{t}} \leq \varepsilon^{\mathrm{T}}\left(\mathrm{A}^{\mathrm{T}}-\mathrm{QQ}^{\mathrm{T}} \mathrm{P}^{-1}\right) \mathrm{P} \varepsilon+\varepsilon^{\mathrm{T}} \mathrm{P}\left(\mathrm{A}-\mathrm{P}^{-1} \mathrm{QQ}^{\mathrm{T}}\right) \varepsilon+\frac{\beta \lambda_{u}}{\alpha-\alpha_{m}} \varepsilon^{T} \varepsilon$

Adding and subtracting the term $2 \alpha \varepsilon^{\mathrm{T}} \mathrm{P} \varepsilon$, and using (20), we get:

$\mathrm{V}_{\mathrm{t}} \leq \varepsilon^{\mathrm{T}}\left(\mathrm{A}^{\mathrm{T}}+\alpha \mathrm{I}\right) \mathrm{P} \varepsilon+\varepsilon^{\mathrm{T}} \mathrm{P}^{\mathrm{T}}(\mathrm{A}+\alpha \mathrm{I}) \varepsilon-2 \varepsilon \mathrm{QQ}^{\mathrm{T}} \varepsilon-2 \alpha \varepsilon^{\mathrm{T}} \varepsilon+\frac{\beta \lambda_{u}}{\alpha-\alpha_{m}} \varepsilon^{T} \varepsilon$

$\mathrm{V}_{\mathrm{t}} \leq \varepsilon \mathrm{QQ}^{\mathrm{T}} \varepsilon-2 \alpha \varepsilon^{\mathrm{T}} \mathrm{P} \varepsilon+\frac{\beta \lambda_{u}}{\alpha-\alpha_{m}} \varepsilon^{T} \varepsilon$

$\mathrm{V}_{\mathrm{t}} \leq-\varepsilon^{+}\left[\lambda_{l}-\frac{\beta \lambda_{u}}{\alpha-\alpha_{m}}\right] \varepsilon^{T} \varepsilon$

Since $\alpha>\alpha+\frac{\beta \lambda_{u}}{(1-\eta) \lambda_{l}}: \lambda_{l}-\frac{\beta \lambda_{u}}{\alpha-\alpha_{m}}>0$

and inequality (21) becomes:

$\mathrm{V}_{\mathrm{t}} \leq \eta \lambda_{1} \varepsilon^{\mathrm{T}} \varepsilon$

Finally, the result follows by making use of the well known Gromwall-Bellman lemma (Khalil, 1996) to obtain:

$\|\varepsilon\|^{2} \leq \gamma_{1}\left\|\varepsilon_{0}\right\|^{2} \mathrm{e}^{-\eta \lambda l t}$

with $\gamma_{1}$ being an arbitrary constant.

Note that condition (19) ensures observability of the states c in system (18) since the observability

Grammian:

$\mathrm{G}=\int_{t}^{t+T} \mathrm{c}(\mathrm{s})^{\mathrm{T}} \mathrm{QQ}^{\mathrm{T}} \mathrm{c}(\mathrm{s}) \mathrm{ds}$

is always positive definite, provided that the number of measurements is larger than, or equal, to the dimension of the reduced order model. In this way, sensor redundancy ensures state reconstruction independently of the particular structure of the dynamical system (18). 


\section{Measurement selection problem}

According to Theorem 1, the performance of the observation schemes can be improved by placing measurements at locations such that the eigenvalue $\lambda_{1}$ in (19) is maximized. This fact suggests the use of the minimum eigenvalue associated to the matrix $\mathrm{QQ}^{\mathrm{T}}$ as a criterion to select, for a given number of sensors $\mathrm{m}$, the appropriate spatial sensor arrangement. Formally, this can be stated as follows:

$$
\operatorname{maxmin}_{P_{m} i=1, k} \lambda_{\mathrm{i}}\left(\mathrm{QQ}^{\mathrm{T}}\right)
$$

Where the operator $\mathrm{P}_{\mathrm{m}}$ indicates the spatial sensor location. This problem could be solved by exhaustive search among all possible $\mathrm{m}$ combinations of the $\mathrm{n}$ coordinates. However, this approach, although feasible in a few number of dimensions, becomes unsuitable for most cases of practical interest due to the high dimensionality of the search space. Alternatively, by taking advantage of the underlying structure of Q, we propose a systematic algorithm to approximate the solution. The approach is based on the following facts:

1) The effect of $P_{m}^{T}$ on $\phi^{\mathrm{T}}$ is that of deleting elements of the basis vectors $\phi_{i}$ at the positions where the columns of $\mathrm{P}_{\mathrm{m}}$ are zero.

2) The scalar products of the resulting sub-vectors $P_{m} \phi_{i}$ are the diagonal elements of $Q Q^{T}$.

3) The eigenvalues of matrix $\mathrm{QQ}^{\mathrm{T}}$ are located inside circles centered at the positions given by the diagonal elements - Gershgorin disc theorem (Golub \& Van Loan, 1983) - with radii satisfying:

$$
r i=\sum_{j \neq i}^{n}\left|\left(Q Q^{T}\right) i j\right|
$$

so that, when the radii are much smaller than the diagonal elements (denoted as $\mathrm{s}_{\mathrm{i}}$ ), problem (22) approximates that of maximizing the minimum diagonal element. Formally, this can be written as:

$$
\max _{P_{m}} \min \left(\mathrm{s}_{1}, \ldots, \mathrm{s}_{\mathrm{k}}\right)
$$

with $\mathrm{s}_{\mathrm{k}}$ being of the form $\mathrm{s}_{\mathrm{k}}=\sum_{j=1}^{m}\left(\phi_{k j}\right)^{2}$

As pointed out in Alonso et al. (2003), the relationship between (22) and (24) holds not only as m approaches $\mathrm{n}$, but also for a given $\mathrm{m}$, as long as $\mathrm{s}_{\mathrm{i}}: \approx \mathrm{s}_{\mathrm{j}}$ for all possible $\mathrm{i}, \mathrm{j}$ elements of the basis set. In this way, the solution to problem (24) can be interpreted as seeking for the "nearest to" orthonormal set of mdimensional sub-vectors $\mathrm{P}_{\mathrm{m}} \phi_{\mathrm{i}}$, thus minimizing orthonormality distortion. A systematic approach to solve problem (24) is presented next.

\subsection{Algorithm description}

Before proceeding with the formal algorithm description, we include the following notation and definitions. 
For each element $\phi_{i} \in R^{n}$ of the set $\mathrm{S}_{\mathrm{k}}$, let us consider the vectors $\sigma_{i}=\left[\left(\phi_{i}\right)_{1}^{2}, \ldots,\left(\phi_{i}\right)_{j}^{2}\right]^{T}, I_{i}=[1: n]$ and an index vector $\eta_{i} \in Z^{m}$ with elements being elements of $\mathrm{I}_{\mathrm{i}}$. In addition, we define the operator $\mathfrak{J}\left(\sigma_{i}, \eta\right)$ as that which computes the summation of elements in $\sigma_{\mathrm{i}}$ with indexes $\left[(\eta)_{1}, \ldots,(\eta)_{\mathrm{m}}\right]^{\mathrm{T}}$. This can be written as: $\Im\left(\sigma_{i}, \eta\right)=\operatorname{sum}\left[\sigma_{i}\left(\eta_{i}\right)\right]$ $\mathrm{s}_{\mathrm{i}}=\mathfrak{J}\left(\sigma_{i}, \eta\right)$

For a given sequence of index vectors $\left\{\eta^{(\mathrm{p})}\right\}$ we also have that:

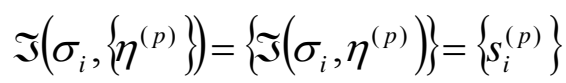

A point $\Sigma$ in the k-summation space $\left(\mathrm{s}_{1}, \ldots, \mathrm{s}_{\mathrm{k}}\right)$ is obtained as:

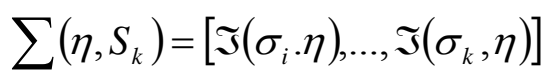

$\sum\left(\eta, S_{k}\right)=\left[s_{1}, \ldots S_{k}\right]$

In the same way, a sequence of points is obtained by applying (25) to a sequence $\left\{\eta^{(p)}\right\}$ so that:

$\sum\left(\left\{\eta^{(p)}\right\}, S_{k}\right)=\left[\mathfrak{I}\left(\sigma_{1},\left\{\eta^{(p)}\right\}\right), \ldots, \mathfrak{I}\left(\sigma_{k},\left\{\eta^{(p)}\right\}\right)\right]$

$\sum\left(\left\{\eta^{(p)}\right\} S_{k}\right)=\left\{\sum\left(\eta^{(p)}, S_{k}\right)=\left\{\sum^{(p)}\right\}\right\}$

A nonincreasing sequence of $\mathrm{r}$ index vectors $\left\{\eta_{i}^{(p)}\right\}$ is defined as that which satisfies:

$\mathfrak{I}\left(\sigma_{i}, \eta_{i}^{(p)}\right) \leq \mathfrak{I}\left(\sigma_{i}, \eta_{i}^{(p-1)}\right)$

for $\mathrm{p}=2, \ldots$, r. Conditional sequences $\left\{\eta_{i}^{(p)}\right\}_{[\mathrm{a}, \mathrm{b}, \mathrm{c}]}$ are defined as nonincreasing sequences which for every $\mathrm{p}$ $=1, \ldots, \mathrm{r}$ satisfy that:

$\sup (\mathrm{a}, \mathrm{c}) \leq \mathfrak{J}\left(\sigma_{i}, \eta_{i}^{(p)}\right) \leq b$

$c \leq \mathfrak{J}\left(\sigma_{j}, \eta_{i}^{(p)}\right)$ for $j=\underset{j \neq i}{1, \ldots, k}$

If either (26) or (27) does not hold for every $\mathrm{p}=1, \ldots, \mathrm{r}$, the sequence is empty i.e. $\left\{\eta_{i}^{(p)}\right\}_{[\mathrm{a}, \mathrm{b}, \mathrm{c}]}=\phi$. A conditional sequence $\left\{\eta_{i}^{(p)}\right\}_{[\mathrm{a}, \mathrm{b}, \mathrm{cc}]}$ is incomplete if there exists some $\eta \notin\left\{\eta_{i}^{(p)}\right\}_{[\mathrm{a}, \mathrm{b}, \mathrm{c}]}$ satisfying both (26) or (27). If not, the conditional sequence is complete. The method employed to compute complete conditional sequences was taken from Alonso et al. (2003). Using these definitions, the algorithm is summarized as follows.

Initialization

Set up positive numbers $\varepsilon, \mathrm{L}^{(0)}$ and $\left\{\bar{L}_{i}\right\}$ for every $\mathrm{i}=1, \ldots, \mathrm{k}$ as $\bar{L}_{i}=\max _{\eta \mathrm{i}} \mathfrak{\Im}\left(\sigma_{i}, \eta_{i}\right)$ 
Iteration Step (from $\ell=0$ )

1) For a given integer $\ell$, set up positive numbers $\mathrm{L}^{(\ell)}$ and define for each i, intervals $\left[L_{i}, \bar{L}_{i}\right]$ with $L_{i}=\bar{L}_{i}-\varepsilon$

2) for each $\mathrm{i}=1, \ldots, \mathrm{k}$.

3) Compute the complete conditional sequence $\left\{\eta_{i}^{(p)}\right\}_{\left.L_{i}, \bar{L}_{i}, L^{(\ell)}\right]}$ (see Alonso et al., 2003) and its corresponding sequence of points $\left\{\sum{ }^{(p)}\right\}_{\left[L_{i}, \bar{L}_{i}, L^{(\ell)}\right]}$

4) Compute

$\left.\mathrm{L}_{\mathrm{i}}=\max _{\mathrm{p}}\left\{\min \left(\sum_{i}^{(p)}\right), \ldots, \min \left(\sum_{i}^{(p)}\right)\right\}\right\}_{\left.L_{i}, \bar{L}_{i}, L^{(\ell)}\right]}$

5) If $\mathrm{L}_{\mathrm{i}}>\mathrm{L}^{(\ell)}$ store current $\mathrm{p}$, set up $\mathrm{L}^{(\ell)}=\mathrm{L}_{\mathrm{i}}$ and go to step 2 for the next $\mathrm{i}$ up to $\mathrm{k}$.

6) If $\mathrm{L}^{(\ell)} \geq \bar{L}_{j}$ for some $\mathrm{j}=1, \ldots, \mathrm{k}$ stop along $\mathrm{j}$.

7) If 6 holds for every $\mathrm{j}=1, \ldots, \mathrm{k}$ save solution $\mathrm{p}, \eta_{i}^{(p)}$ and terminate. If not, set $\mathrm{L}(\ell+1)=\mathrm{L}(\ell), \bar{L}_{i}=L_{i}$ for every $i \neq j$ and go to step 1 with $\ell=\ell+1$.

5. Case study: a nonlinear convection-diffusion-reaction process

In this example, we consider the problem of concentration and temperature reconstruction on a nonisothermal tubular reactor from a limited number of sensors. The system is described by the following set of partial differential equations (Antoniades \& Christofides, 2001b) defined on a spatial domain z $€(0$, 1):

$C_{i}=-\frac{\partial C}{\partial z}+\frac{1}{P e_{C}} \frac{\partial^{2} C}{\partial z^{2}}-f(C, T)$

$T_{i}=-\frac{\partial T}{\partial z}+\frac{1}{P e_{T}} \frac{\partial^{2} T}{\partial z^{2}}+B_{T} f(C, T)+\beta_{T}\left(T_{C}-T\right)$

where $\mathrm{C}$ and $\mathrm{T}$ stand for concentration and temperature, respectively, in deviation form with respect to a stationary state. $T_{c}$ corresponds to the temperature of the cooling medium and $f(C, T)$ represents the reaction term, of the form:

$\mathrm{f}(\mathrm{C} ; \mathrm{T})=\mathrm{B}_{\mathrm{C}}(1+\mathrm{C}) \exp \left[\frac{y T}{1+T}\right]$ 
values:

$\mathrm{Pe}_{\mathrm{C}}=7.0 \quad \mathrm{~B}_{\mathrm{C}}=0.1 \quad \mathrm{y}=10.0$

$\mathrm{Pe}_{\mathrm{T}}=7.0 \quad \mathrm{~B}_{\mathrm{T}}=2.5 \quad \beta_{\mathrm{T}}=2.0$

The reactant at the output stream is assumed to be recycled to the feed stream at a ratio $\mathrm{r}$, which results into the following boundary conditions at $\mathrm{z}=0$ :

$\frac{\partial C}{\partial z}=P e_{C}\left[(1-r) C_{0}+r C(t, 1)-C(t, 0)\right]$

$\frac{\partial T}{\partial z}=P e_{T}\left[(1-r) T_{0}+r T(t, 1)-T(t, 0)\right]$

The corresponding boundary conditions at $\mathrm{z}=1$ are:

$\frac{\partial C}{\partial z}=\frac{\partial T}{\partial z}=0$

Operating at $\mathrm{C}_{0}=\mathrm{T}_{0}=\mathrm{T}_{\mathrm{c}}=0$ with a recycling relation $\mathrm{r}=0.5$, the reactor exhibits an oscillatory behavior as depicted in Fig. 1. The POD basis set is obtained by solving the eigenvalue problem (9) as described in Section 2. The snapshots employed are depicted in Fig. 1(a) and (b) for C and T, respectively. Each snapshot consists of the values of concentration and temperature at a given time taken at 16 equally spaced positions in the domain, and ordered sequentially in a vector $\mathrm{u}=\left(\mathrm{C}^{\mathrm{T}}, \mathrm{T}^{\mathrm{T}}\right)^{\mathrm{T}}$. The energy captured by the first 11 modes - equation (15) - is plotted in Fig. 2. As it can be seen from the Figure, a POD basis set with three elements is enough to collect more than the $99.9 \%$ of the energy thus capturing most of the relevant behavior of the system. The elements of the selected POD basis are depicted in Fig. 3.

For the selected basis set and different numbers of possible given measurements $(\mathrm{m}=6,8,10)$, sensor placements satisfying (24) were computed with the algorithm described in Section 4. Fig. 4 represents, for the different number of sensors available, both the values of the diagonal elements of matrix $\mathrm{QQ}^{\mathrm{T}}$ and their corresponding minimum and maximum eigenvalues obtained as a function of the iteration number. The optimal arrangements (presented in Fig. 5) are compared in Fig. 6, in terms of the maximum and minimum eigenvalues of the resulting $\mathrm{QQ}^{\mathrm{T}}$ matrix, with arrangements obtained through a standard criterion such as the maximization of the trace of the matrix $\mathrm{QQ}^{\mathrm{T}}$ (see Alvarez et al., 1981). As shown in the Figure, the approach suggested in this paper is able to identify sensor arrangements with minimum eigenvalues much larger than those obtained through the standard criterion. In accordance with the discussion in Section 3, this would imply better estimation properties. To confirm this fact, observation experiments were carried out with these two possible arrangements (denoted by A and B, respectively). The system, in these experiments, was perturbed through a fluctuation in the fresh feed stream temperature. The distribution of temperature and concentration under such fluctuation is plotted in Fig. 7 (the class of perturbation introduced in the system is represented in Fig. 8). The evolution of the first (dominant) true c-states - see 
Eq. (2) - and the ones estimated with the proposed observation scheme (17) but different arrangements, are presented in Fig. 8.As illustrated in this Figure, the ability to dynamically observe the dominant modes of the system is to a large extent conditioned by the type of sensor arrangement. In this way, the criterion proposed in this paper induces a very acceptable observer performance as compared with alternative selection criterions.

\section{Conclusions}

In this paper, a systematic technique was presented to efficiently solve the optimal sensor location problem and thus provide reliable field reconstruction from a limited and usually reduced number of measurements. This was accomplished through a two-steps approach. First, a low dimensional representation of the solution of the original distributed system is obtained. Second, the most appropriate sensor type and locations are chosen on this low dimensional sub-space through an efficient guided search algorithm that minimizes orthonormality distortion.

\section{Acknowledgements}

A.A.A. acknowledges the generous support from Xunta de Galicia and Universidade de Vigo; I.G.K. acknowledges support from the National Science Foundation and the AFOSR (Dynamics and Control Program, Dr Marc Jacobs).

\section{References}

Alonso, A. A., Kevrekidis, I., \& Frouzakis, C. (2003). An approach to optimally place sensors for state reconstruction in distributed process systems. American Institute of Chemical Engineering Journal, in press.

Alvarez, J., Romagnoli, J. A., \& Stephanopoulos, G. (1981). Variable measurements structures for the control of a tubular reactor. Chemical Engineering Science 36(10), 1695.

Antoniades, C., \& Christofides, P. D. (2001a). Integrating nonlinear output feedback control and optimal actuator/sensor placement for transport-reaction processes. Chemical Engineering Science 56, 4517.

Antoniades, C., \& Christofides, P. D. (2001b). Studies on nonlinear dynamics and control of a tubular reactor with recycle. Nonlinear Analysis: Theoretical Methods and Applications 47, 5933.

Berkooz, G., Holmes, P., \& Lumley, L. (1993). The proper orthogonal decomposition in the analysis of turbulent flows. Annual Review of Fluid Mechanics 25, 539.

Christofides, P. D., \& Daoutidis, P. (1997). Finite dimensional control of parabolic PDE systems using 
approximate inertial manifolds. Journal of Analytical Applications 2(16), 398.

Courant, R., \& Hilbert, D. (1937). Methods of mathematical physics . New York: Wiley.

Golub, G. H., \& Van Loan, C. (1983). Matrix computations . Baltimore: Johns Hopkins University Press.

Harris, T. J., Wright, J. D., \& MacGregor, J. F. (1980). Optimal sensor location with an application to a packed bed tubular reactor. American Institute of Chemical Engineering Journal 26(6), 910.

Holmes, P., Lumley, J. L., \& Berkooz, G. (1996). Turbulence, coherent structures, dynamical systems and symmetry . Cambridge University Press.

Kammer, D. C. (1991). Optimal sensor placement for model identification using system realization methods. Journal of Guidance and Controlling 19(3), 729.

Keller, J. P., \& Bonvin, D. (1992). Selection of input and output variables as a model reduction problem. Automatica 28(1), 171.

Khalil, H. K. (1996). Nonlinear systems (2nd ed). Upper Saddle River, NJ: Prentice Hall.

Kumar, S., \& Seinfeld, J. H. (1978). Optimal location of measurements for distributed parameter estimation. IEEE Transactions on Automation Control of AC 23(4), 690.

Podvin, B., \& Lumley, J. (1998). Reconstructing the flow in the wall region from wall sensors. Physics of Fluids 10(5), 1182.

Rugh, W. J. (1993). Linear system theory. Englewood Cliffs, NJ: Prentice Hall.

Shvartsman, S. Y., \& Kevrekidis, I. O. (1998). Nonlinear model reduction for control of distributed systems: a computer-assisted study. American Institute of Chemical Engineering Journal 44(7), 1579.

Smoller, J. (1983). Shock waves \& reaction-diffusion equations. New York: Springer.

Van der Berg, F. W. J., Hoefsloot, H. C. J., Boelens, H. F. M., \& Smilde, A. K. (2000). Selection of optimal sensor position in tubular reactor using robust degree of observability criteria. Chemical Engineering Science 55, 827.

Vande Wouwer, A., Point, N., Porteman, S., \& Remy, M. (2000). An approach to the selection of optimal sensor locations in distributed parameter systems. Journal of Process Control 10, 291.

Windes, L. C., Cinar, A., \& Ray, W. H. (1989). Dynamic estimation of temperature and concentration profiles in packed bed reactors. Chemical Engineering Science 44(10), 2087. 
Fig. 1. Snapshots produced by direct numerical simulation of the nonlinear diffusion - convection reaction system - Eqs. (28) and (29). (a) Concentration evolution. (b) Temperature evolution

Fig. 2. Energy captured by the low dimensional set as a function of the number of PODs chosen. Note that three PODs are enough to capture more than $99.9 \%$ of the energy of the system.

Fig. 3. The first three PODs functions chosen in accordance with the energy criterion. (a) Concentration part of the discretized PODs functions. (b) Temperature part of the discretized PODs functions

Fig. 4. Intermediate points (diagonal elements) in the k-summation space (solid lines) and the corresponding maximum and minimum $\mathrm{QQ}^{\mathrm{T}}$ eigenvalues (dotted lines) for the nonlinear convectiondiffusion-reaction example, as a function of the iteration number for different numbers of sensors. (a) $\mathrm{m}=$ 6, (b) $\mathrm{m}=8$, (c) $\mathrm{m}=10$.

Fig. 5. A comparison of the maximum and minimum $\mathrm{QQ}^{\mathrm{T}}$ eigenvalues, for different number of sensors, obtained by: (A) optimal solution of problem (24), (B) maximization of the trace of $\mathrm{QQ}^{\mathrm{T}}$.

Fig. 6. Optimal sensor arrangements for $\mathrm{m}=8$ attained by methods $\mathrm{A}$ and $\mathrm{B}$, respectively. Squares indicate the location of temperature sensors. Triangles indicate the location of concentration sensors

Fig. 7. Concentration and temperature evolution profiles for the nonisothermal chemical reactor in response to temperature fluctuations in the fresh reactant feed stream (the range and shape of the fluctuation is depicted in Fig. 8).

Fig. 8. Evolution of the real and the estimated first three modes under temperature input fluctuation. Measurements are taken through 8 available sensors. Mode estimation is obtained from the observation scheme (17) with gain computed as in Theorem 1. Solid lines represent the evolution of the real modes and modes estimated from sensor arrangements A. Dotted lines represent the evolution of modes for sensor arrangement B. 